Supporing Information

\title{
Ligand Controlled Morphology Evolution of Active Intermediates for the Syntheses of Gold Nanostars
}

\author{
Xianghua Meng, Aravind Baride, and Chaoyang Jiang**
}

Department of Chemistry, University of South Dakota, 414 East Clark Street, Vermillion, South Dakota 57069 USA

* Corresponding author. Fax: (605) 677-6397; E-mail: Chaoyang.Jiang@usd.edu

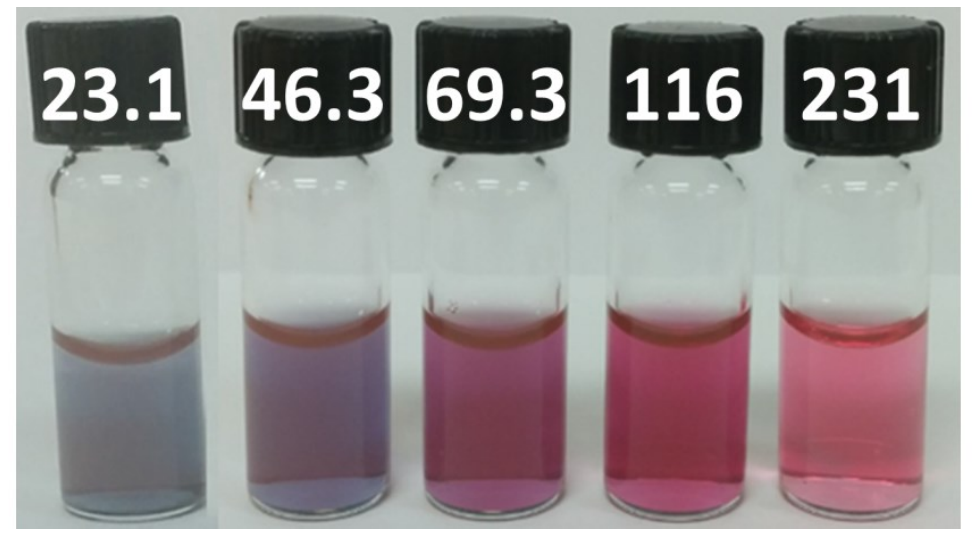

Fig. S1a. Photograph of gold nanostructures prepared with various concentrations of gold nanoparticle seeds (unit of labels: pM). 


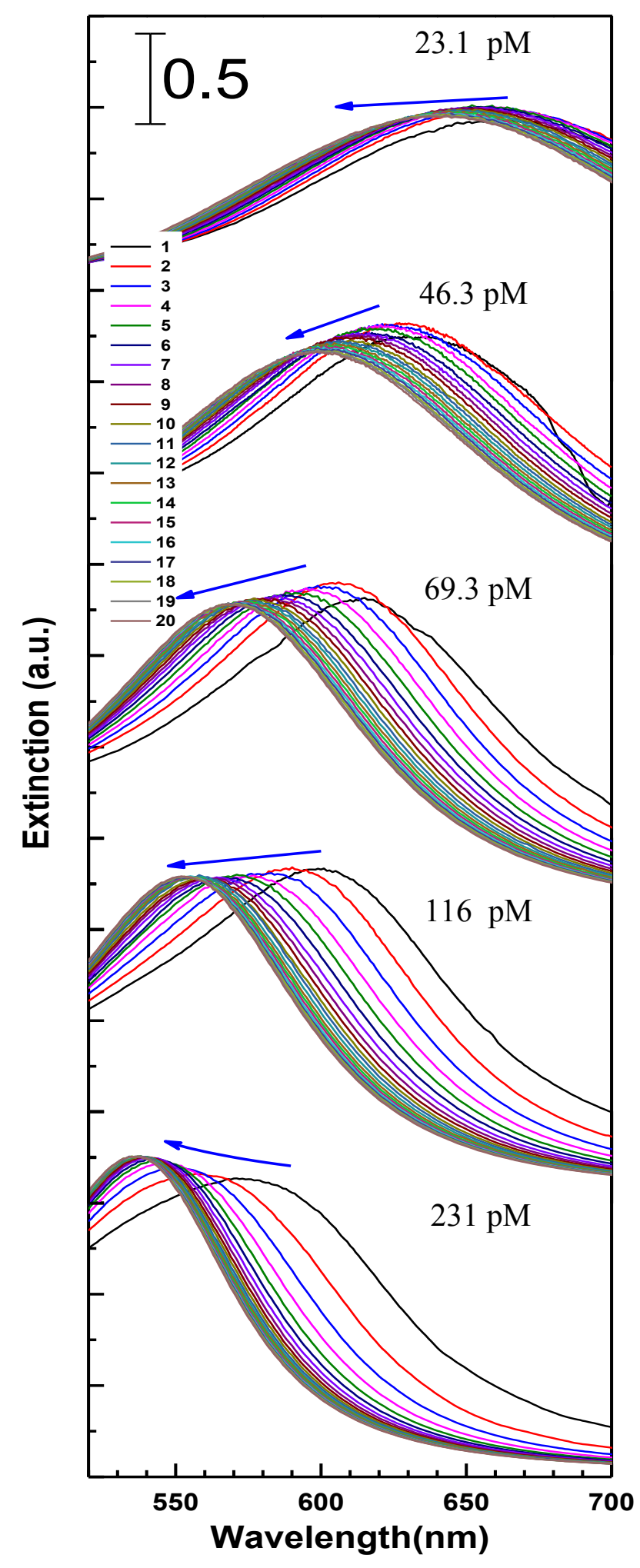

Fig. S1b. in situ UV-vis extinction spectra of reaction during the seed-mediated growth of branched gold nanoparticles with various concentration of gold nanoparticle seeds. 

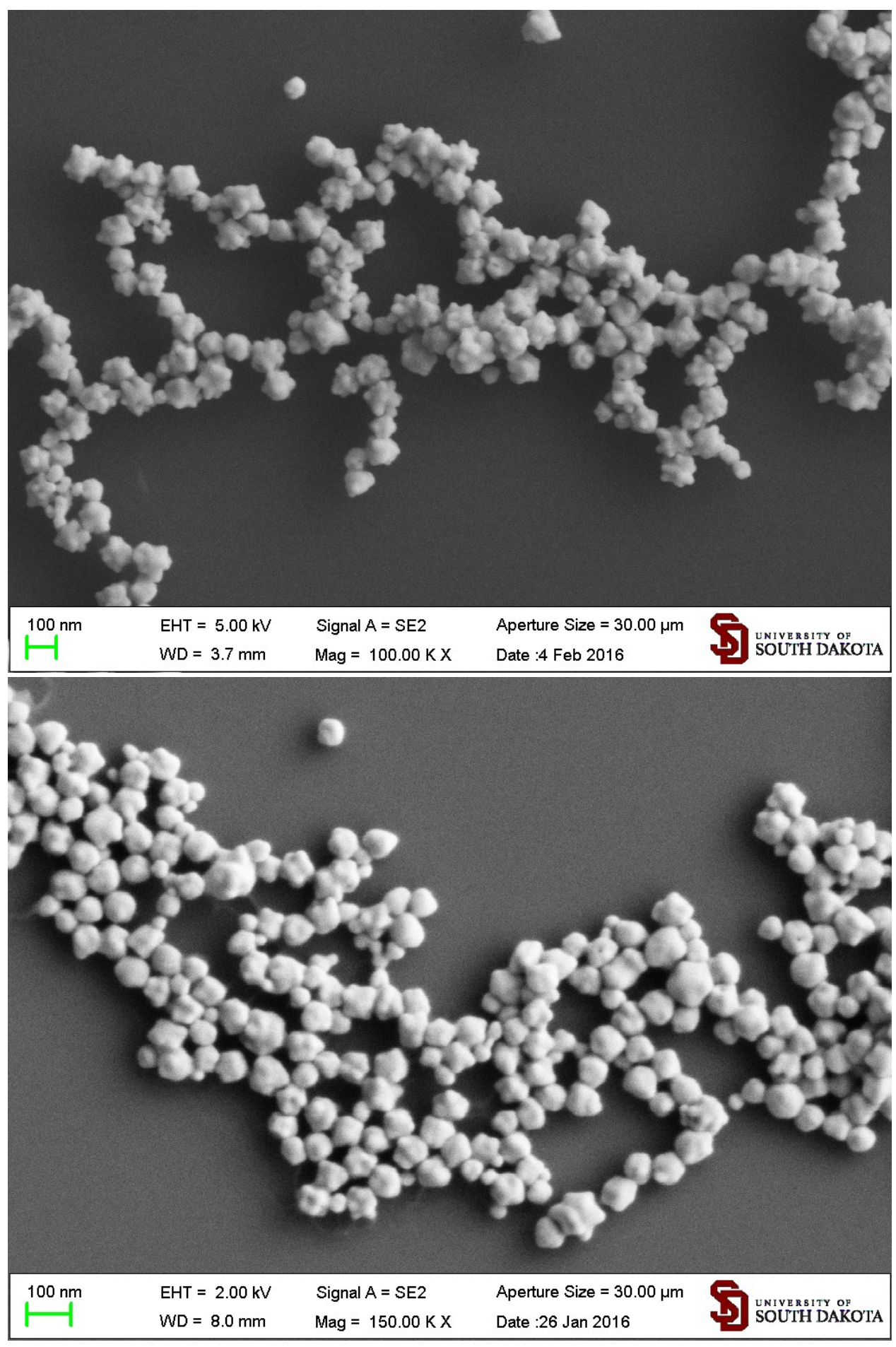

Fig. S2. SEM images of branched gold nanoparticles prepared with different seed concentrations: top: $23.1 \mathrm{pM}$, and bottom: $69.3 \mathrm{pM}$. 


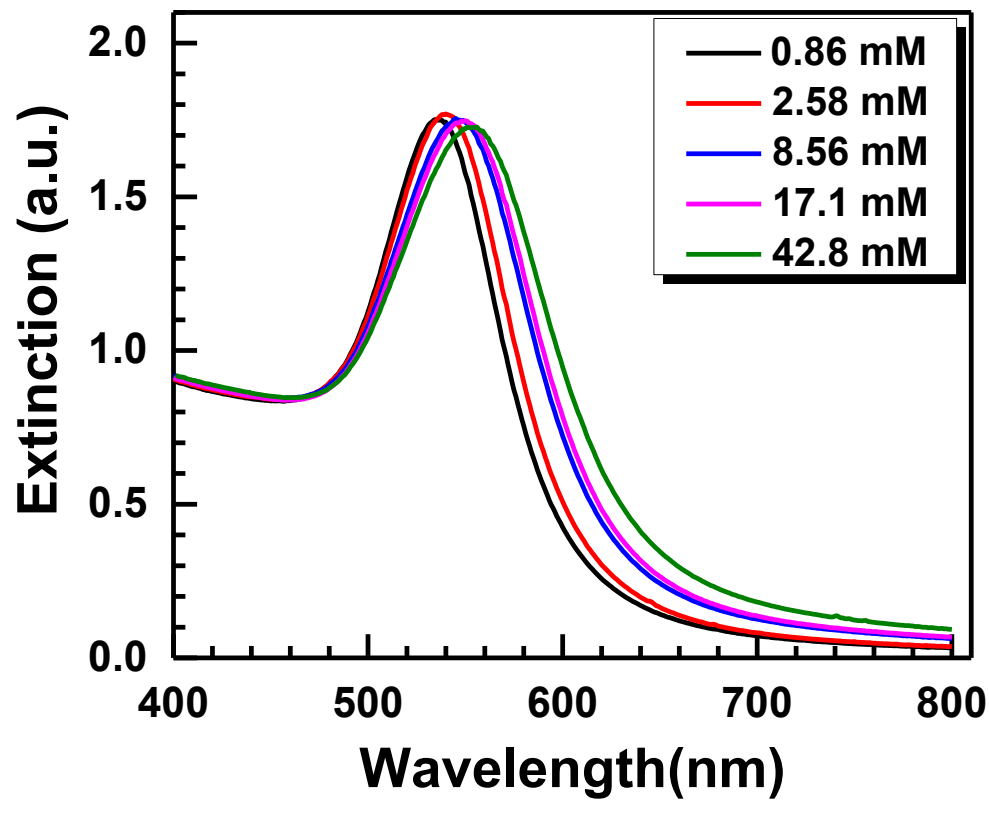

Fig. S3a. in situ UV-vis extinction spectra recorded at 20 minutes of the seed-mediated growth reactions using various concentrations of HQ ligands.

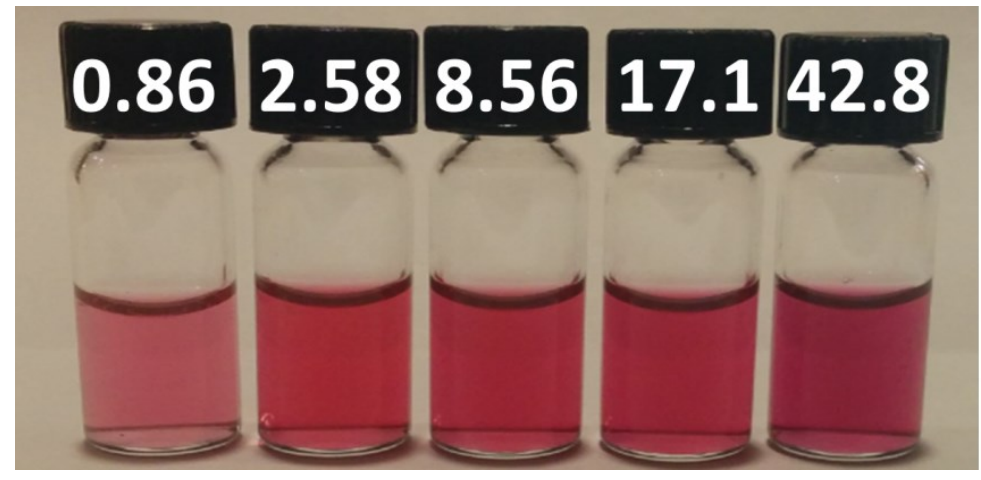

Fig. S3b. Photograph of gold nanostructures prepared with various concentrations of HQ ligands (unit of labels: $\mathrm{mM}$ ). 

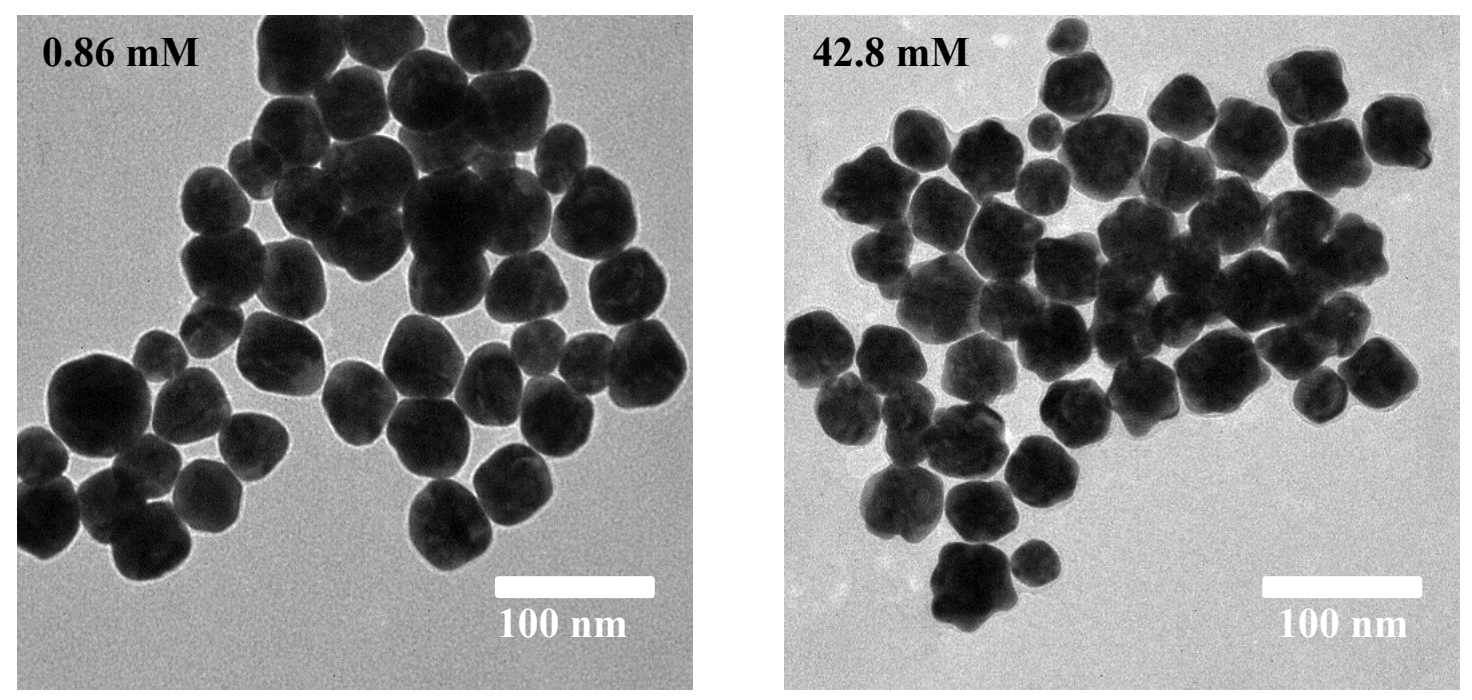

Fig. S3c. TEM images of the resulted branched gold nanoparticles prepared with various concentrations of HQ ligands

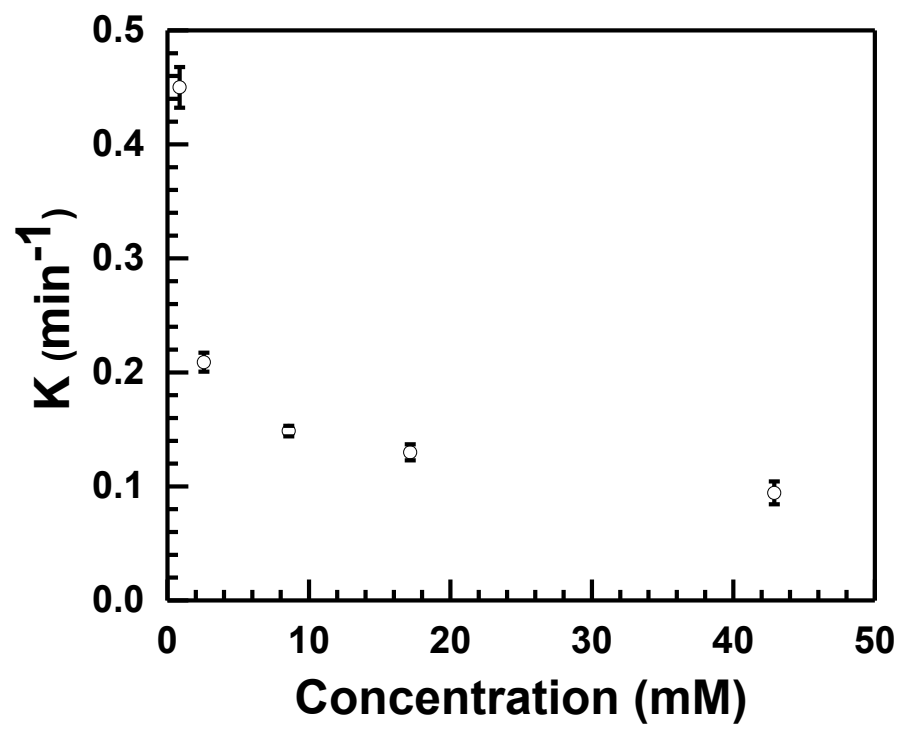

Fig. S4. A plot of rate constant $\mathrm{k}$ for various gold intermediates with different HQ concentrations. 